\title{
Atividade alelopática dos extratos fresco e seco de folhas de barbatimão (Stryphnodendron adstringens (Mart.) Coville) e pata-de- vaca (Bauhinia forficata link) sobre a germinação e desenvolvimento inicial de plântulas de tomate
}

\author{
Allelopathic activity of fresh and dry extracts of Stryphnodendron \\ adstringens (Mart.) Coville and Bauhinia forficata link leaves on the \\ germination and initial development of tomato seedlings
}

\author{
Daniel Donini Manoel ${ }^{1 *}$; Caio Fernando Reis Doiche ${ }^{2}$; \\ Tainara Bortolucci Ferrari ${ }^{3}$; Gisela Ferreira ${ }^{4}$
}

\begin{abstract}
Resumo
O trabalho teve por objetivo verificar a ação alelopática de pata-de-vaca (Bauhinia forficata Link), e barbatimão (Stryphnodendron adstringens (Mart.) Coville) em sementes de tomate (Lycopersicum esculentum Mill). As sementes de tomate foram tratadas com extratos aquosos preparados a partir de folhas frescas e secas das espécies-teste nas concentrações de $100 \%, 75 \%, 50 \%, 25 \%$ e $0 \%$ (controle) e acondicionadas em 'gerbox' transparente. $\mathrm{O}$ delineamento experimental foi inteiramente casualizado, com cinco tratamentos e cinco repetições de 25 sementes por parcela por espécie-teste. Foram avaliados porcentagem, tempo e velocidade médios de germinação e, comprimento médio de raiz primária. Os dados foram submetidos à análise de variância e as médias comparadas por Tukey a $5 \%$ de probabilidade. Não houve diferença em relação à porcentagem de germinação das sementes de tomate para os diferentes tipos de extrato. Em relação à velocidade média de germinação (VMG) todos os tratamentos com extrato foliar fresco diferiram da testemunha. O TMG e a VMG apresentaram diferenças em relação ao extrato de folhas secas nas concentrações $25 \%, 75 \%$ e $100 \%$. Todos os tratamentos apresentaram efeito inibitório sobre o comprimento da raiz primária.
\end{abstract}

Palavras-chave: Alelopatia, sementes, inibição

\begin{abstract}
The objective of this study aimed at the verification of the allelopatic action of extracts of "pata-devaca" (Bauhinia forficata Link) and "barbatimão" (Stryphnodendron adstringens (Mart.) Coville) on seeds of tomato (Lycopersicum esculentum Mill). Tomato seeds placed in translucent "gerbox" were treated with aqueous extracts prepared with fresh and dry leaves of the tested species at the following concentrations: $100 \%, 75 \%, 50 \%, 25 \%$, and $0 \%$ (control). The experimental design was completely randomized with five treatments and five replications of 25 seeds per plot per species. The data were submitted to the analysis of variance and the means compared by Tukey test at $5 \%$ of probability. Germination percentage, mean time and germination rates (MGT and MGR), and mean of the primary
\end{abstract}

\footnotetext{
1 Graduando em Ciências Biológicas. Instituto de Biociências, Unesp, Campus de Botucatu, SP, Brasil. Distrito de Rubião Júnior, s/n. CEP: 18618-000. Caixa postal: 510. Telefone: (14) 3811-6265. E-mail: danieldonini@uol.com.br.

2 Graduando em Engenharia Agronômica, Faculdade de Ciências Agronômicas, Unesp, Botucatu, SP.

3 Doutoranda em Ciências Biológicas (Botânica), Departamento de Botânica, Instituto de Biociências, Unesp, Botucatu, SP.

4 Prof $^{\mathrm{a}}$ Dr $^{\mathrm{a}}$, Departamento de Botânica, Instituto de Biociências, Unesp, Botucatu, SP.

* Autor para correspondência
} 
root length were evaluated. No difference was found for germination percentage of tomato seeds for the different extracts. For MGR, all treatments with fresh leaf extract differed from the check. MGT and MGR showed differences among the following dry leaf extracts concentrations: $25 \%, 75 \%$ and $100 \%$. All treatments presented inhibitory effect on the length of the primary root.

Key words: Allelopathy, seeds, inhibition

\section{Introdução}

A alelopatia é definida como o efeito inibitório ou benéfico, direto ou indireto, de uma planta sobre outra, através da produção de compostos químicos que são liberados no ambiente. Estes compostos, chamados de aleloquímicos ou metabólitos secundários estão presentes em todos os tecidos das plantas, tanto em órgãos vegetativos quanto reprodutivos, sendo que ambos têm potencial para armazenar estes metabólitos (RIZVI et al., 1992; SOUZA et al., 2006). Entretanto, a quantidade e o caminho pelos quais são emitidos diferem de espécie para espécie (FRIEDMAN, 1995). Os aleloquímicos podem ser liberados através de lixiviação, exsudação radicular, volatilização, decomposição de seus resíduos ou outros processos, tanto em sistemas naturais quanto agrícolas (FERGUSON; RATHINASABAPATHI, 2003).

Segundo Rice (1984) após serem liberados por estes processos, os compostos podem causar efeitos diretos e indiretos sobre outras plantas. Efeitos diretos são caracterizados por alterações no metabolismo e crescimento da planta, como no mecanismo de divisão e alongamento celular, rotas hormonais, fotossíntese e respiração. Efeitos indiretos compreendem alterações em propriedades do solo, interferindo na absorção de nutrientes, como também na população e atividade de microrganismos.

Desta maneira, estes efeitos são capazes de influenciar ecossistemas, naturais ou manejados, como os sistemas agrícolas. Comunidades, dominância e sucessão vegetais, tanto quanto manejo e produtividade de culturas, são afetados pelos efeitos alelopáticos de certas espécies, que utilizam este mecanismo de defesa para sua sobrevivência e estabelecimento no local (CHOU, 1986). Sendo assim, várias pesquisas são feitas sobre este mecanismo, utilizando espécies de interesse econômico, como o tomate (CASTRO et al., 1983; GORLA; PEREZ, 1997; GUSMAN; BITTENCOURT; VESTENA, 2008).

A espécie Stryphnodendron adstringens (Mart.) Coville, cujo nome popular é barbatimão, pertence à família Mimosaceae. Ocorre do Cerrado do Pará até São Paulo e Mato Grosso do Sul, em formações primárias ou secundárias. É uma espécie utilizada como ornamental e a madeira em construção civil e marcenaria, sendo também, amplamente empregada como medicinal na maioria das regiões do país. É indicado contra leucorréia, hemorragia, diarréia, hemorróida, para limpeza de ferimento e na forma de gotas contra conjuntivite. O chá da casca é indicado para uso externo em hemorragia uterina, ferida ulcerosa e para pele excessivamente oleosa (FELFILI et al., 1999; LORENZI, 1992; LORENZI; MATOS, 2002).

A espécie Bauhinia forficata Link (pata-de-vaca) pertencente à família Leguminosae, é uma das cerca de 300 espécies do gênero Bauhinia, que apresentase como árvore de porte médio, encontrada do Rio de Janeiro ao Rio Grande do Sul, que possui folhas uncinadas com formato semelhante a pata de vaca, flores brancas e frutos do tipo vagem linear (MARTINS et al., 1998). Algumas espécies do gênero Bauhinia são utilizadas na medicina folclórica da África, Ásia e Américapara o tratamento de várias doenças (ACHENBACH; STOCKER; CONSTENLA, 1988). A infusão das folhas de Bauhina forficata é utilizada na medicina popular brasileira como agente diurético, hipoglicemiante, tônico, depurativo, no combate à elefantíase e na redução da glicosúria (MARTINS et al., 1998). 
Pires et al. (2001) demonstraram a importância de experimentos em laboratório sob condições ótimas de temperatura e umidade, para evidenciar a presença de aleloquímicos em extratos aquosos de tecidos de partes aéreas e das raízes de leucena influenciando a germinação de sementes e desenvolvimento de plântulas de desmódio (Desmodium purpureum), picão preto (Bidens pilosa) e caruru (Amaranthus hybridus).

Sendo assim, o presente trabalho teve como objetivo determinar a existência de atividade alelopática de extratos aquosos das folhas frescas e secas de barbatimão (Stryphnodendron adstringens (Mart.) Coville) e pata-de-vaca (Bauhinia forficata Link) na germinação e no desenvolvimento de plântulas de tomate (Lycopersicum esculentum Mill).

\section{Material e Métodos}

O experimento foi realizado no Laboratório de Germinação do Departamento de Botânica, Instituto de Biociências da Unesp, Botucatu, SP.

Os ensaios foram acondicionados em câmara BOD, com temperatura de $25{ }^{\circ} \mathrm{C} \pm 2{ }^{\circ} \mathrm{C}$. As sementes de tomate foram colocadas em 'gerbox' transparentes, forradas com papel 'germitest'. Para a elaboração do extrato seco as folhas de barbatimão e pata-de-vaca foram coletadas e secadas à $70^{\circ} \mathrm{C}$ em estufa de circulação forçada de ar, enquanto que para a preparação do extrato fresco, as folhas foram coletadas e utilizadas imediatamente. Os extratos foram obtidos triturando-se a parte aérea das espécies em liquidificador durante 1 minuto na proporção de $200 \mathrm{~g}$ para cada litro de água destilada, resultando no extrato bruto, que foi utilizado a $100 \%$. Para os outros tratamentos, houve uma diluição com água destilada para as concentrações de $25 \%, 50 \%$ e $75 \%$. Na testemunha foi utilizada apenas água destilada. $\mathrm{O}$ delineamento experimental foi inteiramente casualizado, com cinco tratamentos e cinco repetições de 25 sementes por parcela.
As avaliações foram realizadas diariamente até que a porcentagem de germinação da testemunha atingisse 90\%. As variáveis avaliadas foram porcentagem de germinação, tempo médio de germinação (TMG), velocidade média de germinação (VMG) e comprimento médio da raiz primária (CMR). Foram consideradas sementes germinadas aquelas que apresentaram raiz primária com aproximadamente dois milímetros de comprimento (HADAS, 1976). O cálculo de TMG foi realizado segundo Edmond e Drapala (1958) e a avaliação de VMG calculada conforme Labouriau (1983).

Os dados obtidos foram submetidos à análise de variância e as médias comparadas pelo teste Tukey a 5\% de probabilidade (PIMENTEL-GOMES, 1990).

\section{Resultdos e Discussão}

As sementes de tomate não apresentaram diferenças significativas para porcentagem de germinação quando tratadas com extrato de folhas frescas, tanto para barbatimão quanto para patade-vaca, em nenhuma concentração utilizada. Para sementes tratadas com extrato de folhas secas foi observada diferença entre extratos das espécies, independentemente das concentrações utilizadas (Tabela 1).

$\mathrm{O}$ fato das concentrações não afetarem a porcentagem de germinação pode ser explicado por Ferreira e Borghetti (2004), os quais relatam que o efeito alelopático não se dá, freqüentemente, sobre a porcentagem de germinação final, e sim sobre a velocidade de germinação ou sobre outro parâmetro, como o comprimento médio de raiz primária. Ferreira e Aqüila (2000) também evidenciaram o fato de a germinação ser menos sensível aos metabólitos secundários do que o crescimento das plântulas, pois este parâmetro é influenciado por estas substâncias que podem levar ao aparecimento de plântulas anormais, tendo a necrose como um sintoma comum. 
Além disso, Chiapusio et al. (1997) indicam que, apesar de a porcentagem de germinação ser um índice muito utilizado, a mesma não evidencia outros aspectos do processo germinativo, por englobar apenas resultados finais, ignorando atrasos ou períodos inativos de germinação durante o bioensaio.

Em relação à velocidade média de germinação (VMG) todas as concentrações do extrato foliar fresco diferiram da testemunha, porém apenas $25 \%$ diferiu em relação ao tipo de extrato, com a maior VMG quando se empregou pata-de-vaca (Tabela 2). Por outro lado, quando empregou-se extrato de barbatimão $(75 \%)$, obteve-se a maior velocidade de germinação $(0,244$ sementes/dia), embora sem diferir do extrato com pata-de-vaca na mesma concentração. Para o extrato preparado com folhas secas de barbatimão pode-se observar diferença na velocidade, em relação à testemunha, quando as sementes foram tratadas com concentrações de $50 \%, 75 \%$ e $100 \%$. Houve decréscimo na velocidade de germinação com o aumento da concentração, embora sem diferenças significativas. Enquanto que o extrato de folhas secas de pata-de-vaca promoveu efeito inibitório em todas as concentrações utilizadas, variando sua intensidade ao aumentar a concentração (Tabela 2).

Tabela 1. Porcentagem média de germinação $(\mathrm{G} \%)$ de sementes de tomate submetidas a tratamento com extratos fresco e seco de barbatimão e pata-de-vaca.

\begin{tabular}{lcc}
\hline \multicolumn{1}{c}{ Tratamentos } & Barbatimão & Pata-de-vaca \\
\hline & & Extrato Fresco \\
Testemunha & 96,0 n.s. & 96,0 n.s. \\
$25 \%$ & 97,0 n.s. & 99,0 n.s. \\
$50 \%$ & 96,0 n.s. & 95,0 n.s. \\
$75 \%$ & 96,0 n.s. & 97,0 n.s. \\
$100 \%$ & 98,0 n.s. & 91,0 n.s. \\
C.V. $(\%)=4,21$ & & \\
\hline
\end{tabular}

C.V. $(\%)=4,21$

Extrato Seco

\begin{tabular}{lcc}
\hline Média & 97,92 a & $95,36 \mathrm{~b}$ \\
\hline C.V. $(\%)=3,678$ & & \\
\hline
\end{tabular}

${ }^{1}$ Valores acompanhados de letras minúsculas iguais não diferem significativamente entres si. Tukey com $\alpha=0,05$

Tabela 2. Velocidade média de germinação $(\mathrm{VMG})$ em sementes de tomate submetidas a tratamento com extratos fresco e seco de barbatimão e pata-de-vaca.

\begin{tabular}{lcccc}
\hline \multicolumn{2}{c}{ VMG (sementes/dia) } \\
\hline \multicolumn{2}{c}{ Estrato Fresco } & \multicolumn{2}{c}{ Estrato Seco } \\
\hline Testemunha $^{1}$ & Barbatimão & Pata-de-vaca & Barbatimão & Pata-de-vaca \\
$25 \%$ & $0,276 \mathrm{Aa}$ & $0,276 \mathrm{Aa}$ & $0,271 \mathrm{Aa}$ & $0,271 \mathrm{Aa}$ \\
$50 \%$ & $0,258 \mathrm{Ba}$ & $0,248 \mathrm{Bb}$ & $0,268 \mathrm{Aa}$ & $0,252 \mathrm{Bb}$ \\
$75 \%$ & $0,254 \mathrm{BCa}$ & $0,248 \mathrm{Ba}$ & $0,253 \mathrm{Ba}$ & $0,249 \mathrm{Ba}$ \\
$100 \%$ & $0,244 \mathrm{Da}$ & $0,247 \mathrm{Ba}$ & $0,252 \mathrm{Ba}$ & $0,237 \mathrm{Cb}$ \\
& $0,248 \mathrm{CDa}$ & $0,246 \mathrm{Ba}$ & $0,247 \mathrm{Ba}$ & $0,238 \mathrm{Db}$ \\
\hline
\end{tabular}

${ }^{1}$ Valores acompanhados de letras iguais, maiúsculas na coluna e minúsculas nas linhas, não diferem significativamente entres si. Tukey $\operatorname{com} \alpha=0,05$. 
Comparando-se os tipos de extrato foliar seco, verifica-se que a pata-de-vaca promoveu a maior VMG na concentração de $100 \%$.

Sobre o tempo médio de germinação (TMG) não foram observadas interações significativas para a germinação de sementes de tomate tratadas com extratos de folhas frescas. Porém, houve diferença entre a testemunha e as demais concentrações independentemente das espécies (Tabela 3) e entre os extratos, independente das concentrações, com a pata-de-vaca promovendo maior TMG (Tabela 4).

Da mesma forma, é observado na Tabela 4 a influência do extrato foliar seco das duas plantas sobre o TMG, sendo que os maiores valores de tempo ocorreram com a utilização dos extratos mais concentrados, o que ratifica os dados de velocidade, uma vez que são inversamente proporcionais. Isto é justificado pelo fato de a velocidade e o tempo estarem correlacionados.

Estes resultados corroboram com o verificado por Piña-Rodrigues e Lopes (2001), que obtiveram a velocidade de germinação reduzida de Tabebuia alba tratada com extrato de Mimosa caesalpinaefolia, porém sem efeito na porcentagem de germinação. De modo semelhante Barreiro, Delachiave e Souza (2005) observaram que extratos de barbatimão não afetaram a porcentagem de germinação de sementes de pepino, porém reduziram significativamente sua velocidade de germinação.

Tabela 3. Tempo médio de germinação (TMG) em sementes de tomate submetidas a concentrações dos extratos de folhas frescas.

\begin{tabular}{lc}
\hline & TMG (dias) \\
\hline Testemunha $^{1}$ & $3,63 \mathrm{C}$ \\
$25 \%$ & $3,95 \mathrm{~B}$ \\
$50 \%$ & $3,98 \mathrm{AB}$ \\
$75 \%$ & $4,07 \mathrm{~A}$ \\
$100 \%$ & $4,04 \mathrm{AB}$ \\
C.V. $(\%)=1,683$ & \\
\hline
\end{tabular}

${ }^{1}$ Valores acompanhados de letras minúsculas iguais não diferem signifcativamente entres si. Tukey com $\alpha=0,05$.

Tabela 4. Tempo médio de germinação (TMG) em sementes de tomate submetidas a tratamento com extratos fresco e seco de barbatimão e pata-de-vaca.

\begin{tabular}{lcc}
\hline \multicolumn{3}{c}{ TMG (dia) } \\
\hline & Barbatimão & Pata-de-vaca \\
\hline Testemunha $^{1}$ & & Extrato Seco \\
$25 \%$ & $3,68 \mathrm{Ba}$ & $3,68 \mathrm{Da}$ \\
$50 \%$ & $3,73 \mathrm{Bb}$ & $3,96 \mathrm{Ca}$ \\
$75 \%$ & $3,94 \mathrm{Aa}$ & $4,01 \mathrm{Ca}$ \\
$100 \%$ & $3,96 \mathrm{Ab}$ & $4,22 \mathrm{Ba}$ \\
C.V. $(\%)=1,925$ & $4,03 \mathrm{Ab}$ & $4,46 \mathrm{Aa}$ \\
\hline
\end{tabular}

Extrato Fresco

\begin{tabular}{lll}
\hline Média & $3,910 \mathrm{~b}$ & $3,954 \mathrm{a}$ \\
\hline C.V. $(\%)=1,683$ & &
\end{tabular}

${ }^{1}$ Valores acompanhados de letras iguais, maiúsculas na coluna e minúsculas nas linhas, não diferem significativamente entres si. Tukey $\operatorname{com} \alpha=0,05$. 
Em relação ao comprimento médio de raiz primária(CMR)em plântulas de tomate, foiobservada atividade alelopática inibitória dos extratos fresco e seco. Verificou-se redução do CMR, comparado ao controle, em todas as concentrações, havendo maior redução com o aumento da concentração, tanto para o barbatimão quanto para a pata-de-vaca (Tabela 5).

Observou-se o mesmo efeito em trabalhos de Gatti, Perez e Lima (2004) em qual plântulas de alface e rabanete tiveram seu comprimento radicular significativamente reduzidos ao serem tratados com extratos de folhas, caules ou raízes de Aristolochia esperanzae O. Kuntze.

Da mesma forma, a inibição em plântulas de alface tratadas com extrato de várias plantas nativas, porém sem redução significativa da parte aérea, também foi verificada por Maraschin-Silva e Áquila (2006). Chung, Ahn e Yun (2001) explicam o efeito inibitório mais acentuado sobre as raízes devido ao contato mais íntimo destas com a solução do aleloquímico, em relação à parte aérea.

Tabela 5. Comprimento médio de raiz (CMR) em sementes de tomate submetidas a tratamento com extratos fresco e seco de barbatimão e pata-de-vaca.

\begin{tabular}{lcccc}
\hline \multicolumn{3}{c}{ CMR (cm) } \\
\hline \multicolumn{2}{c}{ Estrato Fresco } & \multicolumn{2}{c}{ Estrato Seco } \\
\hline Testemunha $^{1}$ & Barbatimão & Pata-de-vaca & Barbatimão & Pata-de-vaca \\
$25 \%$ & $7,85 \mathrm{Aa}$ & $7,85 \mathrm{Aa}$ & $7,34 \mathrm{Aa}$ & $7,34 \mathrm{Aa}$ \\
$50 \%$ & $3,82 \mathrm{Ba}$ & $2,22 \mathrm{Bb}$ & $5,60 \mathrm{Ba}$ & $3,02 \mathrm{Bb}$ \\
$75 \%$ & $2,82 \mathrm{Ca}$ & $2,37 \mathrm{Ba}$ & $3,64 \mathrm{Ca}$ & $3,36 \mathrm{Ba}$ \\
$100 \%$ & $1,92 \mathrm{Da}$ & $1,87 \mathrm{Ba}$ & $2,98 \mathrm{Ca}$ & $2,14 \mathrm{BCa}$ \\
& $1,07 \mathrm{~Eb}$ & $2,02 \mathrm{Ba}$ & $2,92 \mathrm{Ca}$ & $1,14 \mathrm{Cb}$ \\
\hline
\end{tabular}

${ }^{1}$ Valores acompanhados de letras iguais, maiúsculas na coluna e minúsculas nas linhas, não diferem significativamente entres si. Tukey com $\alpha=0,05$.

\section{Conclusão}

Conclui-se que extratos de folhas frescas e secas de barbatimão e pata-de-vaca apresentaram efeito alelopático sobre tempo, velocidade médios de germinação e comprimento médio de raiz de tomate, sendo este efeito mais efetivo nas concentrações mais elevadas com extrato foliar fresco de barbatimão e extrato foliar seco de pata-de-vaca.

\section{Referências}

ACHENBACH, H.; STOCKER, M.; CONSTENLA, M. A. Flavonoids and other constituents of Bauhinia manca. Phytochemistry, Inglaterra, v. 27, n. 6, p. 1835-1841, 1988.

BARREIRO, A. P.; DELACHIAVE, M. E. A.; SOUZA, F. S. Efeito alelopático de extratos de parte aérea de barbatimão (Stryphnodendron adstringens (Mart.) Coville) na germinação e desenvolvimento da plântula de pepino. Revista Brasileira de Plantas Medicinais, Botucatu, v. 8, n. 1, p. 4-8, 2005.

CASTRO, P. R. C.; RODRÍGUEZ, J. D.; MORAES, M. A.; CARVALHO, V. L. M. Efeitos alelopáticos de alguns extratos vegetais na germinação do tomateiro (Lycopersicum esculentum Mill. cv. Santa Cruz). Planta Daninha, Jaboticabal, v. 6, n. 2, p. 79-85, 1983.

CHIAPUSIO, G.; SÁNCHEZ, A. M.; REIGOSA, M. J.; GONZÁLEZ, L.; PELLISSIER, L. Do germination indices adequately reflect allelochemical effects on the germination process? Journal of Chemical Ecology, Nova York, v. 23, n. 11, p. 2445-2453, 1997.

CHOU, C. The role of allelopathy in subtropical agroecosystems in Taiwan. In: PUTNAM, A.; TANG, C. (Ed.). The Science of Allelopathy. New York: John Wiley, 1986. p. 57-73. 
CHUNG, I. M.; AHN, J. K., YUN, S. J. Assesment of allelopathic potential of barnyard grass (Echinochloa crus-gall) on rice (Oriza sativa L.) cultivars. Crop Protection, Guildford, v. 20, n. 10, p. 921-928, 2001.

EDMOND, J. D.; DRAPALA, W. J. The effects of temperature, sand and soil, and acetone on germination of okra seeds. Proceedings of the American Society Horticultural Science, Alexandria, v. 71, n. 2, p. 428-434, 1958.

FELFILI, J. M.; SILVA JUNIOR, M. C.; DIAS, B. J.; REZENDE, A. V. Estudo fenológico de Stryphnodendron adstringens (Mart.) Coville no cerrado sensu stricto da Fazenda Água Limpa no Distrito Federal, Brasil. Revista Brasileira de Botânica, São Paulo, v. 22, n. 1, p. 83-90, 1999.

FERGUSON, J. J.; RATHINASABAPATHI, B. Allelopathy: how plants suppress other plants. Florida: Ed. da University of Florida, 2003.

FERREIRA, A. G.; AQUILA, M. E. A. Alelopatia: uma área emergente da ecofisiologia vegetal. Revista Brasileira de Fisiologia Vegetal, São Paulo, v. 12, n. 1, p. 175-204, 2000.

FERREIRA, A. G.; BORGHETTI, F. Germinação: do básico ao aplicado. Porto Alegre: Artmed, 2004.

FRIEDMAN, J. Allelopathy, autotoxicity, and germination. In: KIGEL, J.; GALILI, G. (Ed.). Seed development and germination. New York: Marcel Dekker, 1995. p. 629-644.

GATTI, A. B.; PEREZ, S. C. J. G. A.; LIMA, M. I. S. Atividade alelopática de extratos aquosos de Aristolochia esperanzae $\mathrm{O}$. Kuntze na germinação e no crescimento de Lactuca sativa L. e Raphanus sativus L. Acta Botânica Brasílica, São Paulo, v. 18, n. 3, p. 459-472, 2004.

GORLA, C. M.; PEREZ, S. C. J. G. A. Influência de extratos aquosos de folhas de Miconia albicans Triana, Lantana camara L., Leucaena leucocephala (Lam) de Wit e Drimys winteri Forst, na germinação e crescimento inicial de sementes de tomate e pepino. Revista Brasileira de Sementes, Pelotas, v. 19, n. 2, p. 260-266, 1997.

GUSMAN, G. S.; BITTENCOURT, A. H. C.; VESTENA, S. Alelopatia de Baccharis dracunculifolia DC. sobre a germinação e desenvolvimento de espécies cultivadas. Acta Scientiarum. Biological Sciences, Maringá, v. 30, n. 2, p. 119-125, 2008.
HADAS, A. Water uptake and germination of leguminous seeds under changing external water potential in osmotic solution. Journal of Experimental Botany, London, v. 27, n. 98 , p. 480-489, 1976.

LABOURIAU, L. G. A Germinação das sementes. Washington: Organização dos Estados Americanos, 1983.

LORENZI, H. Árvores brasileiras: manual de nativas do Brasil. Nova Odessa: Plantarum, 1992.

LORENZI, H.; MATOS, F. J. A. Plantas medicinais no Brasil: nativas e exóticas cultivadas. Nova Odessa: Instituto Plantarum, 2002.

MARASCHIN-SILVA, F.; AQUILA, M. E. A. Potencial alelopático de espécies nativas na germinação e crescimento inicial de Lactuca sativa L. (Asteraceae). Acta Botânica Brasílica, São Paulo, v. 20, n. 1, p. 61-69, 2006.

MARTINS, R. E.; CASTRO, D. M.; CASTELLANI, D. C.; DIAS, J. E. Plantas Medicinais. Viçosa: Ed. da UFV, 1998.

PIMENTEL-GOMES, F. Curso de estatística experimental. 13. ed. Piracicaba: Nobel, 1990.

PIÑA-RODRIGUES, F. C. M; LOPES, B. M. Potencial alelopático de Mimosa caesalpinaefolia Benth sobre sementes de Tabebuia alba (Cham.) Sandw. Floresta e Ambiente, Rio de Janeiro, v. 8, n. 1, p. 130-136, 2001.

PIRES, N. M.; PRATES, H. T.; PEREIRA-FILHO, I. A; OLIVEIRA, R. S.; FARIA, T. C. L. Atividade alelopática da leucena sobre espécies da plantas daninhas. Sciencia Agricola, Piracicaba, v. 58, n. 1, p. 61-65, 2001.

RICE, E. L. Allelopathy. Orlando: Academic Press, 1984.

RIZVI, S. J. H.; HAQUE, H.; SINGH, U. K.; RIZVI, V. A discipline called allelopathy. In: RIZVI, S. J. H.; RIZVI, H. (Ed.). Allelopathy: Basic and applied aspects. London: Chapman \& Hall, 1992. p. 1-10.

SOUZA, L. S.; VELINI, E. D.; MARTINS, D.; ROSOLEM, C. A. Efeito alelopático de capim-braquiária (Brachiaria decumbens) sobre o crescimento inicial de sete espécies de plantas cultivadas. Planta Daninha, Viçosa, v. 24, n. 4, p. 657-668, 2006. 
